\title{
Size effect characteristics of structural surfaces by improved projective covering method
}

\author{
S. J. Chen ${ }^{1 *}$, , J. P. Chang ${ }^{1}$, X. G. Liu ${ }^{2}$ and Z. H. Li ${ }^{1}$
}

*Correspondence:
chenshijiang_2003@163.com
${ }^{1}$ Institute of Mining
Research, Inner Mongolia
University of Science
and Technology, Baotou,
Inner Mongolia 014010,
People's Republic of China
Full list of author information
is available at the end of the
article

*Correspondence: chenshijiang_2003@163.com and Technology, Baotou Inner Mongolia 014010, article

\begin{abstract}
Accurate characteristic of structural surfaces roughness at the relevant scale is very important to understand mechanical properties of rock mass discontinuities. So, a systematic investigation has been carried out to understand the effect of scale on the structural surface roughness by fractal dimension method. Firstly, considering the shortcoming of the projective covering method (PCM), we improved this method based on stochastic approach. Secondly, to investigate the size effect of the structural surface roughness, we selected six sampling windows, respectively, from the central and four corners part of structural planes $(2 \mathrm{~m} \times 2 \mathrm{~m})$. The sampling windows range from $62.5 \mathrm{~mm} \times 62.5 \mathrm{~mm}$ to $2000 \mathrm{~mm} \times 2000 \mathrm{~mm}$. And then, we calculated fractal parameters of the different size surfaces using improved projective covering method (IPCM) at the same resolution. Thirdly, we discussed a new method of determining reasonable size of structural surfaces by the parameter $\Delta D_{\max }^{S D}$. This parameter is difference of the maximum fractal dimension of the same size structural surface in different regions. The results show that: (1) The size effect of structure surfaces is different with different morphological surface. Generally, as the size increases, the roughness of structure surfaces increases and then decreases. There is positive size effect in small scale and negative size effect in large scale. (2) For a given structural surface, when the same size surfaces are selected from different locations of the structural planes, and the size effect characteristics are also different. (3) As the size of structure surfaces increases, the parameter $\Delta D_{\max }^{S D}$ gradually decreases and tends to almost constant. The result of this study is a useful supplement to the comprehensive understanding of the size effect of structural surfaces roughness.
\end{abstract}

Keywords: Structural surfaces, Size effect; fractal dimensions, Projective covering method, Stochastic analysis

\section{Introduction}

Discontinuities such as cracks, faults, fractures and joints always exist in rocks as a result of various geological processes [21]. Moreover, strength, deformability and flow properties of these discontinuities are strongly affected not only by their surface morphology but also size scale [16]. Therefore, investigating size effect of surface 
morphology by accurate description method is a critically important to understand discontinuities mechanics characterization.

Since Barton and Bandis [3] introduced the scale effect of rock structural surfaces, the scholars in related fields have carried out discussions on this issue and formed three academic views. (1) Some scholars believe that as the size of structural surfaces increases, its roughness will decrease, which is the negative scale effect of structural surfaces $[2,6,8,10,12]$. (2) Some scholars believe that as the size of structural surfaces increases, its roughness also increases, namely the positive scale effect $[11,18]$. (3) Others believe that there is no scale effect on roughness of structural surfaces [23]. The possible reasons for these three different views are the size and morphological characteristics of investigated structural surfaces, as well as using differences description methods. In addition, the measurement resolution is another reason for different views about understanding size effect of structure surfaces [26]. Based on the above analysis, comprehensive understanding size effect of structure surfaces still need to study.

The size effect of structural surfaces is mostly studied by the morphological characterization parameters. At present, these methods describing structure surfaces can be categorized broadly into Barton typical contour method, statistical parameters, and fractal dimensions [13]. Among them, the statistical parameters mainly include fluctuation amplitude parameters $[22,27]$ : mean square value $(M S)$, root mean square $(R M S)$, central line average $(C L A)$, first derivative root mean square $\left(Z_{2}\right)$, second derivative root mean square $\left(Z_{3}\right)$, average fluctuation amplitude parameters $\left(Z_{4}\right)$, autocorrelation function $(A C F)$, structure function $(S F)$, Angle parameters $[4,14,29]$ : standard deviation of undulation Angle $(S D)$, The mean positive inclination angle of the profile $\left(S_{p+}\right)$, The mean negative inclination angle of the profile $\left(S_{p-}\right)$, The mean inclination angle for the surface $\left(\theta_{\mathrm{s}}\right)$, The maximum apparent dip angle in the shear $\operatorname{direction}\left(\theta_{\mathrm{Max}} / \mathrm{C}+1\right)$, The roughness profiles or surfaces index $[6,9,13]$ : the ratio of actual length of a roughness profile to its projected length on the horizontal surface $\left(R_{p}\right)$, the ratio of actual area of a roughness surface to its projected area on the horizontal surface $(R s)$, bright area percentage $(B A P)$ and quantifying joint surfaces anisotropic behavior by Variogram Approach $(S R v)$. The aforementioned parameters, statistical parameters describing the roughness of structural surfaces is influenced by sampling interval [15, 25, 28, 30]. And the Barton typical contour method has the shortcomings of subjective judgment. Although the fractal dimension description method is also controversial, its calculation process is substantially different from the other methods, and has more advantages.

The fractal method for describing $3 \mathrm{~d}$ structural surfaces are mainly included as following: Triangular prism surface area $\operatorname{method}(T P S A M)$ [7], Projective covering $\operatorname{method}(P C M)$ [32, 31], Modification of projective covering method(MPCM) [17], Cube covering method $(C C M)$ [33], Improved cubic covering method $(I C C M)$ [19, 20, 34], Differential cubic covering method $(D C C M)$ and relative differential cubic covering $\operatorname{method}(R D C C M)$ [1]. Among the above-mentioned methods of direct measurement, the PCM is an early proposed method for estimating the roughness of structural surfaces, and it is a very effective method for quantitative description of $3 \mathrm{~d}$ structural surfaces. But, there is a small defect in calculating the area of grid cell about PCM. So, based on stochastic analysis, we proposed improved projective covering method (IPCM). 


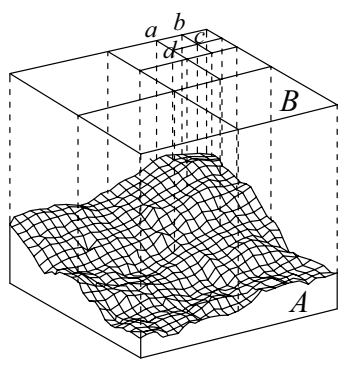

(a)

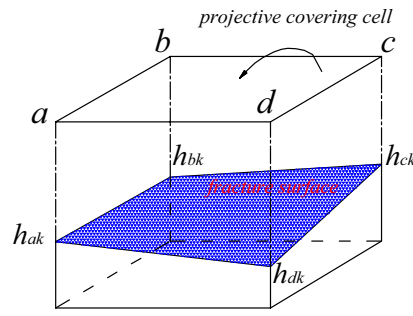

(b)

Fig. 1 The projective covering method

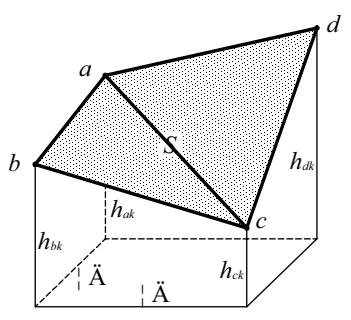

(a)

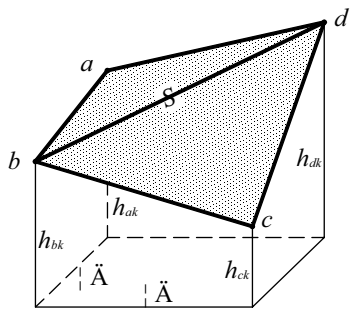

(b)

Fig. 2 Two schemes of triangulation of an elementary surface

In this paper, using the IPCM, we investigated size effect of the large size structural surfaces $(2 \mathrm{~m} \times 2 \mathrm{~m})$. And, we proposed a method using the fractal parameter $\Delta D_{\max }^{S D}$ to estimate reasonable size of structural surfaces for laboratory test. The parameter $\Delta D_{\max }^{S D}$ means the maximum dimension difference of the same size structure surfaces but from different regions. This research provides a new approach for understanding comprehensively the size effect characteristics of structural surfaces.

\section{Algorithms and steps of the IPCM}

\section{The shortcoming of the PCM}

In the PCM method, the analyzed surface is divided into a lot of small grid cells and the area of rough surface surrounded by points $\boldsymbol{a b c d}$ (see Fig. 1) can be approximately calculated by [31]

$$
\begin{aligned}
A_{k}(\delta)= & \frac{1}{2}\left\{\left[\delta^{2}+\left(h_{a k}-h_{d k}\right)^{2}\right]^{1 / 2}\left[\delta^{2}+\left(h_{d k}-h_{c k}\right)^{2}\right]^{1 / 2}\right. \\
& \left.+\left[\delta^{2}+\left(h_{a k}-h_{b k}\right)^{2}\right]^{1 / 2}\left[\delta^{2}+\left(h_{b k}-h_{c k}\right)^{2}\right]^{1 / 2}\right\}
\end{aligned}
$$

Here, $A_{k}(\delta)$ is the area of the kth cell square; the $h_{a k}, h_{b k}, h_{c k}$ and $h_{d k}$ are the heights of a fracture surface at points $a, b, c$ and $d$, respectively; the $\delta$ means step size.

Following the Eq. 1, in fact, each area of the grid cell is calculated as sum of two triangles. Unfortunately, the triangle areas of grid cells are not accurately calculated, which 
was pointed out by Kwaśny [17]. So, the author modified the PCM by introducing a more precise area calculation method. Nevertheless, the area of the fracture surface is still approximate because there are two ways to divide the field surrounded by four points on fracture surfaces into two triangles (as shown in Fig. 2). In this way, the small grid cell is divided by different triangulation division scheme, and the calculated fractal dimension is different, which has been confirmed [33].

In order to overcome the above shortcoming, the random number is introduced into the PCM. Specifically speaking, a random connection method is adopted to determine triangulation division scheme of each grid cell. So, the millions of grid cells in whole structural surfaces are more consistent with the actual morphology. This fractal dimension method expressing structural surfaces is called Improved projective covering method (IPCM).

\section{Algorithms of the IPCM}

In fractal geometry, the measure of a fractal object in E-dimensional space can be expressed in a general form [31]

$$
G(\delta)=G_{0} \delta^{E-D}
$$

where $E$ represents Euclidean dimension. This equation can be used for the measurement of a fractal object in a form of either curve, area or volume. For instance, if $E=2, G$ and $\delta$ in Eq. 2 correspond to a fractal area, then Eq. 2 yields

$$
A_{T}(\delta)=A_{T 0} \delta^{2-D}
$$

where $A_{T 0}$ denotes the apparent area of the rough surface. $A_{T}(\delta)$ means the entire area of the rough surface under a certain scale measurement and it can be expressed by

$$
A_{T}(\delta)=\sum_{i=1}^{N(\delta)} A_{i}(\delta)
$$

where $N(\delta)$ is the total number of cells with scale of $\delta \times \delta$ needed to cover the rough surface.

From Eqs. 3 and 4, we have the following relation

$$
D=2-\frac{\ln \left[\sum_{i=1}^{N(\delta)} A_{i}(\delta) / A_{T 0}\right]}{\ln (\delta)}=2-\beta
$$

where $\beta$ is the slope of the $\ln -\ln$ plot $A_{T}(\delta)$ vs $\delta$.

\section{Analysis steps of the IPCM}

(1) We obtain the three-dimensional topography data of the studied fracture surface. As far as obtaining methods, there are the digital image processing (DIP) method and $3 \mathrm{~d}$ laser scanning method.

(2) We divide the fracture surface into small grid cells with the step size $\delta$. 
(3) We determine triangulation division scheme of each grid cell by random connection method and calculate the area. Specifically speaking, a random number is generated by the random function and the parity of the random number is determined. If the number is odd, the area of the small grid cell is calculated using the triangular connection scheme of Fig. 2a. Otherwise, the area of the grid cell is calculated by the triangular connection scheme of Fig. 2b.

(4) Given a step size $\delta$, the total area of the fracture surface is calculated with the Eq. 4. And then, calculate point pairs $\left\{\operatorname{In}\left(A_{r}(\delta) / A_{r 0}\right), \operatorname{In}(\delta)\right\}$.

(5) The procedure (2)-(4) is repeated for each different step size. We can calculate the value of the different points $\left\{\operatorname{In}\left(A_{r}(\delta) / A_{r 0}\right), \operatorname{In}(\delta)\right\}$ at different $\delta \times \delta$ scale. And then, the slope $\beta$ is fitted by the least square method. Thus, the fractal dimension $(D)$ can be calculated by Eq. 5 .

(6) Perform repetition calculations according to above steps (2)-(5) and form a sample space of fractal dimension. And then, the fractal dimension samples are tested by distribution fitting method. When the test results show that the sample data belong to some probability distribution, the samples mean is taken as the exact value of the fractal dimension of the studied structural plane. Otherwise, the number of samples will be increased and the sample distribution fit test will be repeated.

The IPCM method calculation program is shown in Fig. 3.

\section{Fractal analysis of the synthetic surface by IPCM}

In this paper, using IPCM, we investigate characteristics of the structural plane generated by Buzzi [5] based on the measured joint section. The grayscale images of the synthetic surface are shown in Fig. 4. The size of the synthetic surfaces both are $2000 \mathrm{~mm} \times 2000 \mathrm{~mm}$. In order to satisfy the accurate calculation of fractal dimension, they are expressed as 2049 pixels $\times 2049$ pixels.

Through the measurement of the gray image in Fig. $4 \mathrm{a}$ and b, the difference between the maximum gray value and the minimum are $[251,0]$ and $[255,0]$, respectively. In fact, the difference between the highest point and the lowest are $160 \mathrm{~mm}$ and $180 \mathrm{~mm}$, respectively. Therefore, the ratio 160/251 and 180/255 are used for fractal calculation of the synthetic surfaces image.

Firstly, according to the procedure of IPCM method, we calculated the synthetic surface in Fig. 4a, and analyzed the calculation results by IPCM and PCM, respectively. Secondly, on this basis, we proposed the simple program for fractal dimension. Finally, for two synthetic surfaces in Fig. 4, we calculated the fractal dimension by simple program of IPCM and analyzed the size effect characteristics.

\section{Fractal calculation and analysis of the synthetic surface \\ Obtained fractal dimension samples}

According to the calculation steps of the Fig. 3, the fractal dimension $(D)$ of the synthetic surface in Fig. 4a is calculated and 100 samples of $D$ are obtained. For each calculation, the scale space is $2 \mathrm{~mm}-32 \mathrm{~mm}$. The 100 samples of $D$ of the synthetic surface are shown in the Table 1. 


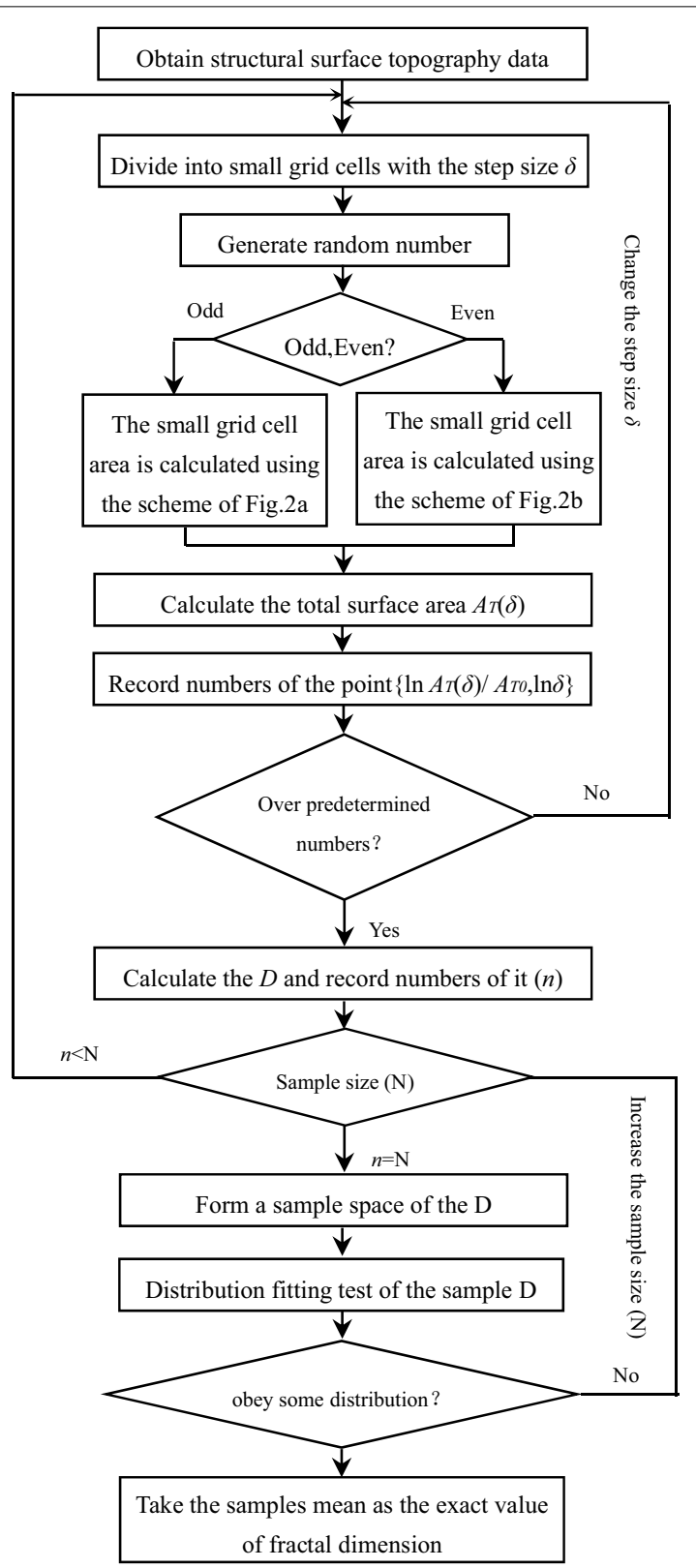

Fig. 3 Design procedure of IPCM method

\section{Distribution characteristics of the fractal dimension samples}

The fractal dimension samples of the synthetic surface are tested based on the probabilistic and mathematical statistics method [24].

According to the data in Table 1, we know, the minimum and maximum fractal dimensions are 2.197516 and 2.197755 , respectively. Now, we may set a range, which is $[2.1975155,2.1977555]$. And then, the range is divided into 8 subsets and the each distance of the adjacent subsets is 0.00003 . The statistical parameters of the subsets (such as interval, counts, frequency) are shown in Table 2. 


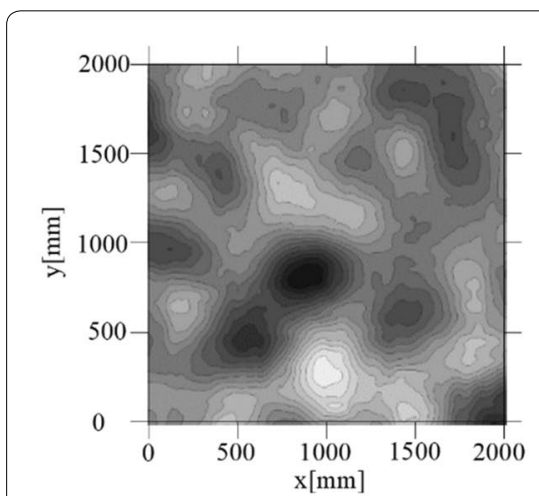

(a)

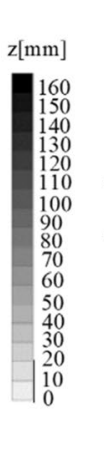

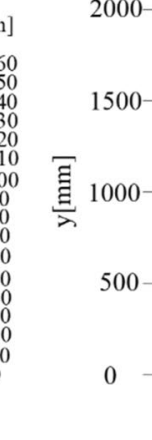

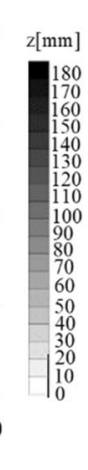

(b)

Fig. 4 The synthetic surfaces by random field model

Table 1100 Samples of fractal dimensions of the synthetic surfaces

\begin{tabular}{llllllllll}
\hline No & $\boldsymbol{D}$ value & No & $\boldsymbol{D}$ value & No & $\boldsymbol{D}$ value & No & $\boldsymbol{D}$ value & No & $\boldsymbol{D}$ value \\
\hline 1 & 2.197656 & 21 & 2.197632 & 41 & 2.197730 & 61 & 2.197516 & 81 & 2.197657 \\
2 & 2.197577 & 22 & 2.197538 & 42 & 2.197724 & 62 & 2.197709 & 82 & 2.197750 \\
3 & 2.197576 & 23 & 2.197630 & 43 & 2.197650 & 63 & 2.197685 & 83 & 2.197686 \\
4 & 2.197615 & 24 & 2.197641 & 44 & 2.197656 & 64 & 2.197662 & 84 & 2.197519 \\
5 & 2.197649 & 25 & 2.197651 & 45 & 2.197728 & 65 & 2.197681 & 85 & 2.197624 \\
6 & 2.197675 & 26 & 2.197733 & 46 & 2.197663 & 66 & 2.197614 & 86 & 2.197675 \\
7 & 2.197545 & 27 & 2.197584 & 47 & 2.197679 & 67 & 2.197602 & 87 & 2.197621 \\
8 & 2.197621 & 28 & 2.197743 & 48 & 2.197611 & 68 & 2.197755 & 88 & 2.197671 \\
9 & 2.197610 & 29 & 2.197603 & 49 & 2.197716 & 69 & 2.197611 & 89 & 2.197715 \\
10 & 2.197718 & 30 & 2.197566 & 50 & 2.197665 & 70 & 2.197727 & 90 & 2.197660 \\
11 & 2.197603 & 31 & 2.197599 & 51 & 2.197654 & 71 & 2.197612 & 91 & 2.197610 \\
12 & 2.197745 & 32 & 2.197628 & 52 & 2.197702 & 72 & 2.197569 & 92 & 2.197663 \\
13 & 2.197660 & 33 & 2.197678 & 53 & 2.197610 & 73 & 2.197651 & 93 & 2.197666 \\
14 & 2.197582 & 34 & 2.197637 & 54 & 2.197608 & 74 & 2.197691 & 94 & 2.197588 \\
15 & 2.197544 & 35 & 2.197600 & 55 & 2.197631 & 75 & 2.197657 & 95 & 2.197675 \\
16 & 2.197590 & 36 & 2.197729 & 56 & 2.197743 & 76 & 2.197691 & 96 & 2.197639 \\
17 & 2.197530 & 37 & 2.197625 & 57 & 2.197686 & 77 & 2.197634 & 97 & 2.197585 \\
18 & 2.197592 & 38 & 2.197607 & 58 & 2.197589 & 78 & 2.197634 & 98 & 2.197642 \\
19 & 2.197711 & 39 & 2.197729 & 59 & 2.197679 & 79 & 2.197590 & 99 & 2.197718 \\
20 & 2.197640 & 40 & 2.197615 & 60 & 2.197628 & 80 & 2.197607 & 100 & 2.197576 \\
\hline
\end{tabular}

Table 2 Statistical parameters of the samples of fractal dimensions

\begin{tabular}{lllllllll}
\hline No & $\mathbf{1}$ & $\mathbf{2}$ & $\mathbf{3}$ & $\mathbf{4}$ & $\mathbf{5}$ & $\mathbf{6}$ & $\mathbf{7}$ & $\mathbf{8}$ \\
\hline Subsets & $2.1975155-$ & $2.1975455-$ & $2.1975755-$ & $2.1976055-$ & $2.1976355-$ & $2.1976655-$ & $2.1976955-$ & $2.1977255-$ \\
& 2.1975455 & 2.1975755 & 2.1976055 & 2.1976355 & 2.1976655 & 2.1976955 & 2.1977255 & 2.1977555 \\
Counts $f_{i}$ & 6 & 2 & 16 & 23 & 20 & 14 & 8 & 11 \\
$\begin{array}{l}\text { Frequency } \\
f_{i} / \mathrm{n}\end{array}$ & 0.06 & 0.02 & 0.16 & 0.23 & 0.20 & 0.14 & 0.08 & 0.11 \\
\hline
\end{tabular}




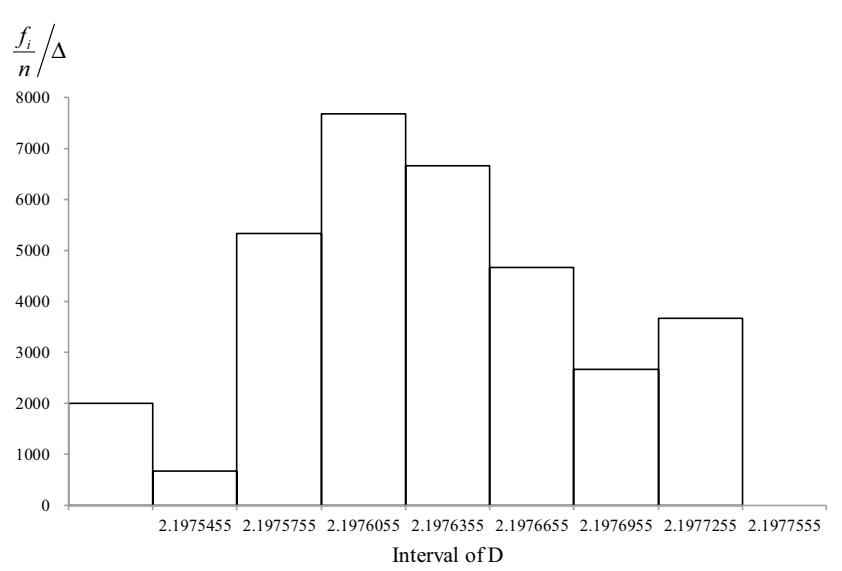

Fig. 5 Histograph of 100 samples of fractal dimensions

According to the data in Table 2, a small rectangle with $\frac{f_{i}}{n} / \Delta$ height is made in each small subset. Where $f_{i}$ is the counts of samples in the $i$ th subset, $n$ is the sample size and $\Delta$ is the distance of the adjacent subsets. In this way, we can get the distribution of fractal dimension samples, as shown in Fig. 5.

From the histogram of Fig. 5, the center section is high and both sides are low. The shape of distribution of the fractal dimension samples is approximate symmetry. So, we can preliminarily judge the samples belongs to the Normal distribution. Now, the $\chi^{2}$ method is used for testing and the statistical variable $\chi^{2}$ is expressed by

$$
\chi^{2}=\sum_{i=1}^{k} \frac{f_{i}^{2}}{n \hat{p}_{i}}-n
$$

where $k$ is the number of sample subsets, and $\hat{p}_{i}$ is the probability estimation of the $i$ th subset.

Hypothesis testing:

$H_{0}$ : The probability density of $X$ is

$$
f(x)=\frac{1}{\sqrt{2 \pi} \sigma} e^{-\frac{(x-\mu)}{2 \sigma^{2}}},-\infty<x<+\infty
$$

where $\mu$ and $\sigma$ are the mean and variance of the samples, respectively, which is unknown in this hypothesis testing. In this example, the estimation value of $\mu$ and $\sigma$ are 2.197644 and 0.000056 by the maximum likelihood estimation method, respectively. If $H_{0}$ is true, the probability density of $X$ is expressed by,

$$
\hat{f}(x)=\frac{1}{\sqrt{2 \pi} \times 0.000056} e^{-\frac{(x-2.197644)}{2 \times 0.000056^{2}}},-\infty<x<+\infty
$$

Now, we define probability event as $A_{i}$. Here, $i$ equals to 8. According to Eqs. 6 and 7 and the Standard Normal Distribution Function Table [24], we can get the estimated value of probability $P\left(A_{i}\right)$. The results are shown in Table 3 . Here, the parameter $n \hat{p}_{i}$ 


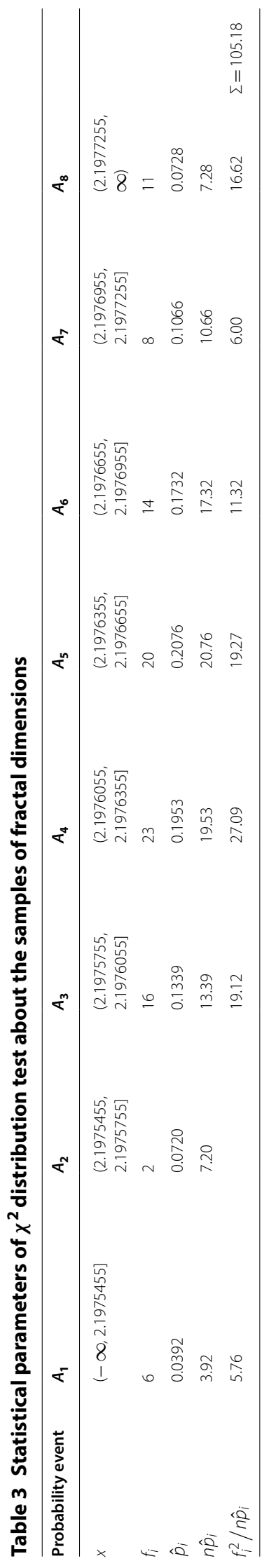




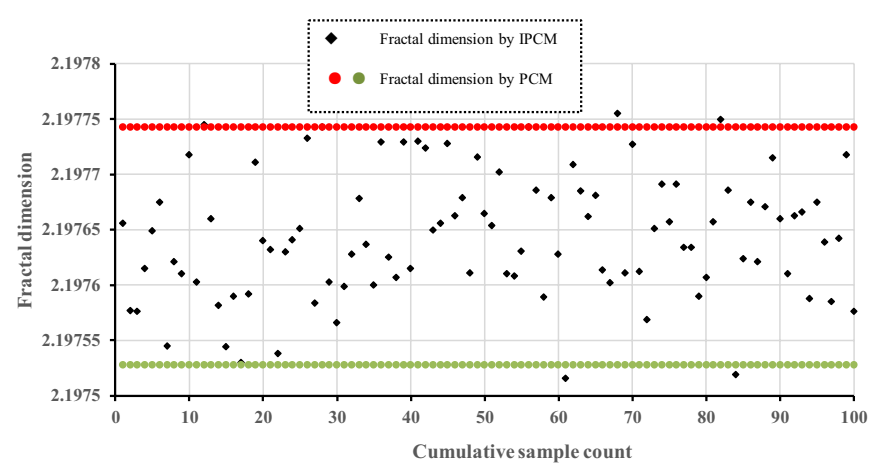

Fig. 6 Comparison IPCM with PCM

in Table 3 should be no less than 5 [24]. Otherwise, the adjacent subsets $A_{i}$ should be properly merged.

According to Eq. $6, \chi^{2}=105.18-100=5.18 . \chi_{0.05}^{2}(k-r-1)=\chi_{0.05}^{2}(4)=9.488>5.18$. Here, $k$ is the number of valid subsets and it equals to $7, r$ means degrees of freedom and it equals to 2. Therefore, we accept $H_{0}$ at level 0.05 . From the above analysis, we can consider that the fractal dimension data are derived from the Normal distribution.

\section{Determination of exact fractal dimension value of the synthetic surface}

From the above analysis, the samples of the fractal dimension of the synthetic surface belong to the Normal distribution. Now, we can take the mean value of the samples as the exact fractal dimension of the synthetic surface. As thus, the exact fractal dimension of the synthetic surface is calculated as 2.197644 .

\section{Comparison IPCM with PCM}

In order to investigate characteristics of fractal dimensions of structural surfaces obtained by the IPCM and PCM respectively, first, we calculated fractal dimension of the synthetic surface by PCM. This method has two connection modes as shown in Fig. 2. So, the calculation results are 2.197743 and 2.197528 , respectively. And then, we

\section{Table 4 Statistical parameter mean from a few samples}

\begin{tabular}{ll}
\hline No & Mean \\
\hline 1 & 2.1976242 \\
2 & 2.1976197 \\
3 & 2.1976321 \\
4 & 2.1976447 \\
5 & 2.1976822 \\
6 & 2.1976530 \\
7 & 2.1976562 \\
8 & 2.1976336 \\
9 & 2.1976578 \\
10 & 2.1976362
\end{tabular}




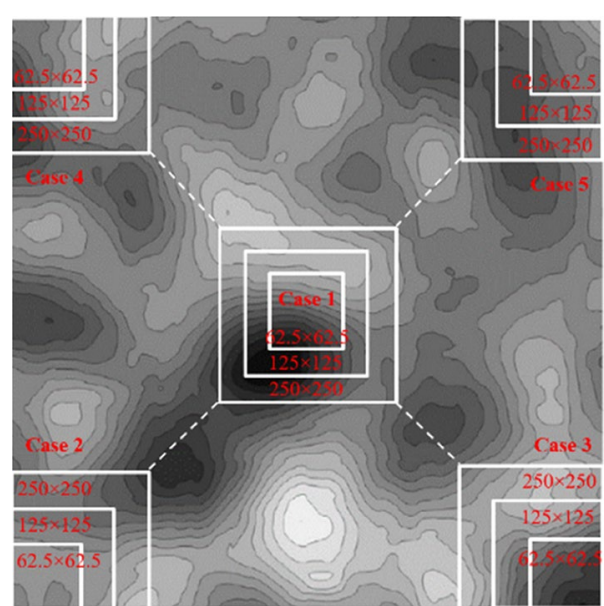

Fig. 7 Division scheme of varisized structure surface

Table 5 Fractal dimensions for samples with different size at five schemes

\begin{tabular}{|c|c|c|c|c|c|c|c|}
\hline Size $(\mathrm{mm} \times \mathrm{mm})$ & & $2000 \times 2000$ & $1000 \times 1000$ & $500 \times 500$ & $250 \times 250$ & $125 \times 125$ & $62.5 \times 62.5$ \\
\hline & Case 1 & 2.197624 & 2.179174 & 2.192371 & 2.245221 & 2.276409 & 2.313967 \\
\hline & Case 2 & 2.197624 & 2.208777 & 2.164691 & 2.130095 & 2.158039 & 2.152421 \\
\hline \multirow[t]{3}{*}{ Figure $3 a$} & Case 3 & 2.197624 & 2.230114 & 2.242755 & 2.179879 & 2.064291 & 2.062817 \\
\hline & Case 4 & 2.197624 & 2.189423 & 2.189950 & 2.197605 & 2.171516 & 2.154560 \\
\hline & Case 5 & 2.197624 & 2.156866 & 2.144126 & 2.165214 & 2.178987 & 2.186229 \\
\hline$D_{A}^{S D}$ & & 2.197624 & 2.192871 & 2.186779 & 2.183603 & 2.169848 & 2.173999 \\
\hline \multirow[t]{3}{*}{$\Delta D_{\max }^{S D}$} & & & 0.073248 & 0.098629 & 0.115126 & 0.212118 & 0.251150 \\
\hline & Case 1 & 2.447770 & 2.452522 & 2.450057 & 2.463435 & 2.444199 & 2.382865 \\
\hline & Case 2 & 2.447770 & 2.458964 & 2.475679 & 2.456437 & 2.388016 & 2.365948 \\
\hline \multirow[t]{3}{*}{ Figure 3b } & Case 3 & 2.447770 & 2.442293 & 2.443463 & 2.438059 & 2.444947 & 2.377290 \\
\hline & Case 4 & 2.447770 & 2.448461 & 2.453102 & 2.466008 & 2.398627 & 2.364124 \\
\hline & Case 5 & 2.447770 & 2.440806 & 2.438959 & 2.453740 & 2.456107 & 2.431264 \\
\hline$D_{A}^{S D}$ & & 2.447770 & 2.448609 & 2.452252 & 2.455536 & 2.426379 & 2.384298 \\
\hline$\Delta D_{\max }^{S D}$ & & & 0.073248 & 0.036720 & 0.027949 & 0.068091 & 0.06714 \\
\hline
\end{tabular}

take the results by PCM and the data in Table 1 which were obtained through IPCM to be presented in the form of a graph, as shown in Fig. 6. Form Fig. 6, we can see, comparing with results by IPCM, the calculation values by PCM are almost the maximum and minimum. Since random numbers are involved in the calculation of IPCM, the results of fractal dimension of structural surface obtained by this method is a series of data. And, this data is subject to normal distribution law from the previous section analysis. Therefore, compared with results by PCM, it is more accurate to use the average value through IPCM as the fractal dimension for quantifing structural surface roughness. 


\section{A simple program of IPCM for fractal dimension}

Considering probability, by random connection method, the thousands of small grid cells in the whole structure plane are more consistent with the actual morphology. From the calculation results as shown in Fig. 6, the fractal dimension by PCM is almost the limit value. So, we think that it will be more reasonable that the mean of fractal dimension samples by IPCM is taken as the accurate fractal dimension of structure surfaces.

However, from the above analysis process of the IPCM, to estimate fractal dimension of fracture surfaces is gruelling and time-consuming work. Whether it is possible, with the mean of a few samples, to regard as the result of structural surfaces by fractal dimension is still to be discussed. In this paper, 100 datum in Table 1 are divided into 10 groups in sequence. And then, the mean of each group is calculated, as shown in Table 4.

If the mean of 100 samples can be considered as the exact values of the fractal dimension of the studied structure surface, and then there are 10 groups which the calculation accuracy of each mean can achieve 0.0001 . From the above results, we can

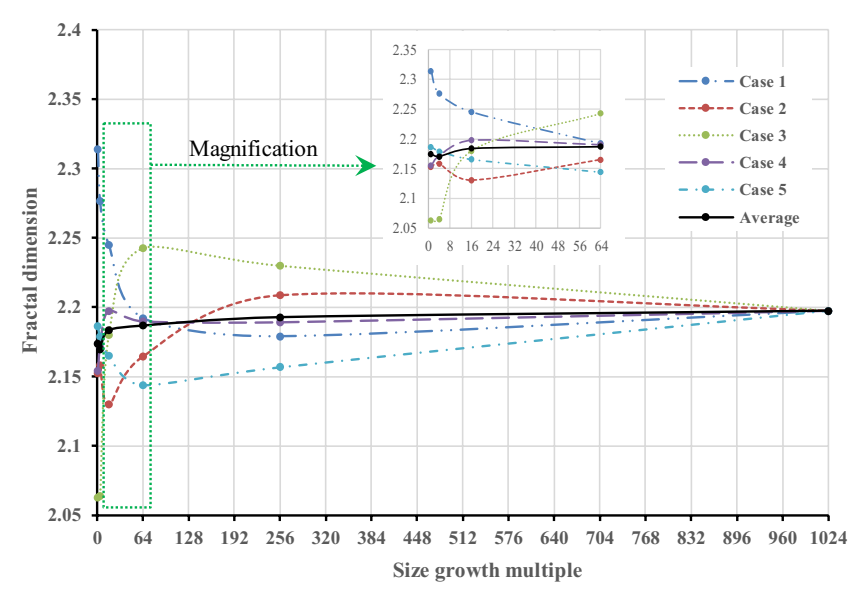

(a) The map of changing trend for Fig.4a

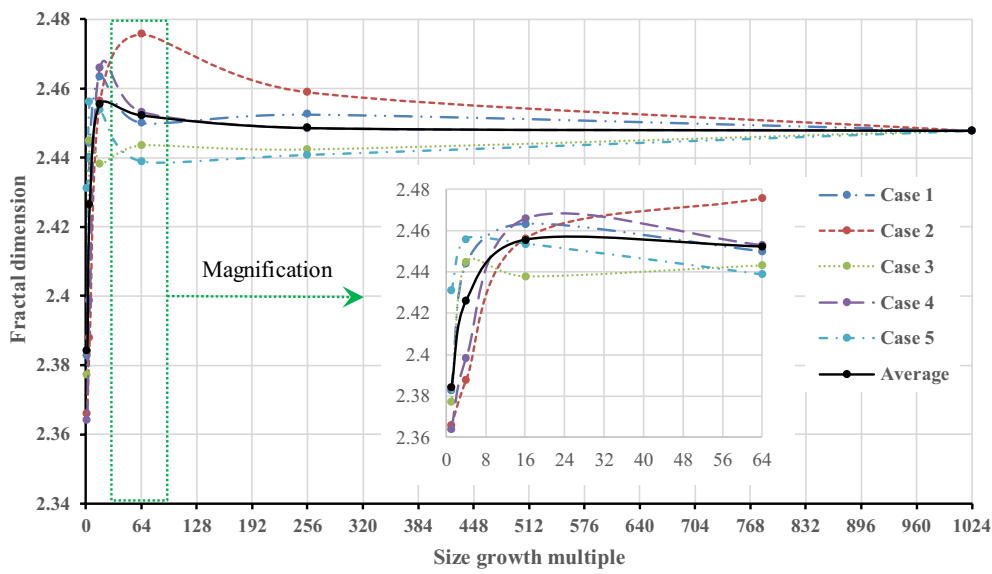

(b) The map of changing trend for Fig.4b

Fig. 8 The relationship between the fractal dimension and the size growth multiple at five schemes 
use the mean of a few samples to represent fractal dimension of structural surfaces when satisfying a certain accuracy requirement. In this way, compared with the more samples, it can save many time and effort. So, it is also feasible to use the mean of a few samples to represent the fractal dimension of structural planes.

\section{Application of IPCM in the size effect of structural surfaces}

\section{Division scheme for structure surfaces}

In this paper, we take the random model structural surface [5] as the researched object for the size effect analysis, as shown Fig. 4. Figure $4 \mathrm{a}$ and $\mathrm{b}$ are obviously different in their morphological features. Comparatively speaking, the asperity distribution about Fig. 4a structural surface is uneven and that of Fig. 4b is uniform. In order to investigate size effect of the structural surfaces, we divide the each surface into six small surfaces and their size (pixel $\times$ pixel) are $65 \times 65,129 \times 129,257 \times 257$, $513 \times 513,1025 \times 1025,2049 \times 2049$ respectively. In addition, we choose five representative schemes as shown in Fig. 7. In order to obtain the data of the specified range structure surface, we developed data reading program.

\section{Fractal dimension calculation and results analysis}

According to IPCM method, we calculated the fractal dimension of different size structure surfaces with different area. The fractal dimension of each structure surface is calculated 10 times and the average is regarded as the result of fractal dimension. The results of calculation fractal dimension are shown in Table 5. In the Table 5, the parameter $D_{A}^{S D}$ means average of fractal dimensions of the same size structure surface but from different area, and the parameter $\Delta D_{\max }^{S D}$ means the maximum difference of fractal dimensions of the same size structure surfaces but from different area.

Here, the $62.5 \mathrm{~mm} \times 62.5 \mathrm{~mm}$ surface is taken as reference plane. And then, the 125 $\mathrm{mm} \times 125 \mathrm{~mm} 、 250 \mathrm{~mm} \times 250 \mathrm{~mm} 、 500 \mathrm{~mm} \times 500 \mathrm{~mm} 、 1000 \mathrm{~mm} \times 1000 \mathrm{~mm} 、 2$ $000 \mathrm{~mm} \times 2000 \mathrm{~mm}$ surfaces are as 4 times, 16 times, 64 times, 256 times and 1024 times, respectively, as $62.5 \mathrm{~mm} \times 62.5 \mathrm{~mm}$ surface. Now, we take size growth factor as the horizontal axis, and establish the relationship between the sizes and fractal dimensions as shown in Fig. 8.

From Fig. 8, for the different morphological feature surfaces, there are different trends of the parameter $D_{A}^{S D}$ with the size increase. To the Fig. 4a structural surface,

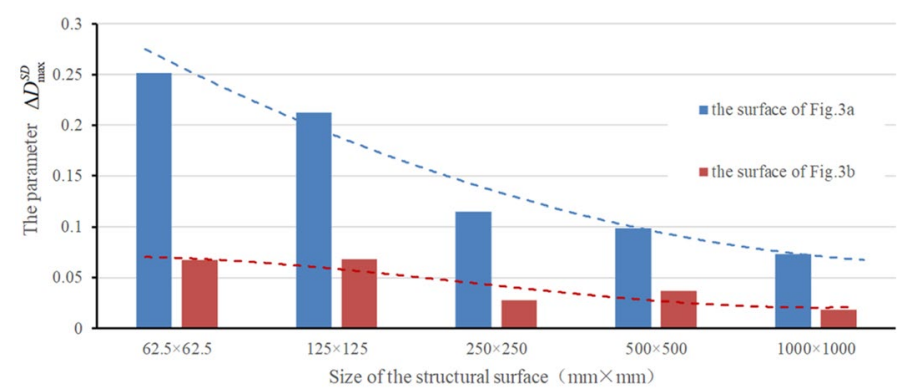

Fig. 9 The relationship between the parameter and the size of the sampling window 
its concavo-convex body is sparse and unevenly distributed, and corresponding, the trend of the parameter $D_{A}^{S D}$ rises with the size increase on the whole. While, to the Fig. 4b structural surface, its concavo-convex body is dense and equally distributed, accordingly, the trend of the parameter $D_{A}^{S D}$ rises and then reduces to the stable value.

In addition, to the same structure surface but different division schemes, there are different trends of fractal dimensions with the size increase. Now, we take the Fig. 4a structural surface for example. About Case 1, the trend of the fractal dimensions declines and gradually tend to the stable value as the structure surface size increases. However, about Case 2, the trend of the fractal dimensions goes up and gradually tend to the stable value with the size increases.

It can be seen from the above phenomenon that the structure surface with complex morphology has both positive and negative size effect characteristics. This size effect rule can be understood as follows. To any structural surface, we can find a small smooth plane with a small fractal dimension. As the size of the structural plane increases, some complex morphology is gradually included, and the fractal dimension is also increasing. This is the positive size effect of the structural plane. When the structure surface continues to increase, the roughness information of the structure surface does not increase, on the contrary, some smooth information maybe is included. So, the fractal dimension will be decreasing. This is the negative size effect of the structure surface. For a regular structural plane, no matter how big, it contains the same morphological information. Therefore, the fractal dimensions for different size surfaces are stable on the whole. For an example, about the very smooth plane, its fractal dimensions are always 2 at any size. This is the dimensionless effect characteristic of structural planes. According to the above analysis, the size effect of structural surfaces is related to the morphological characteristics.

\section{A new method to determine reasonable size of structural surfaces}

If the fractal dimensions expressing two surfaces which are the same size but from different area are close to each other, we can conclude that morphological characteristics of the structural surfaces are generally consistent. Based on this understanding, we can use the parameter $\Delta D_{\max }^{S D}$ to determine the reasonable size of structural planes.

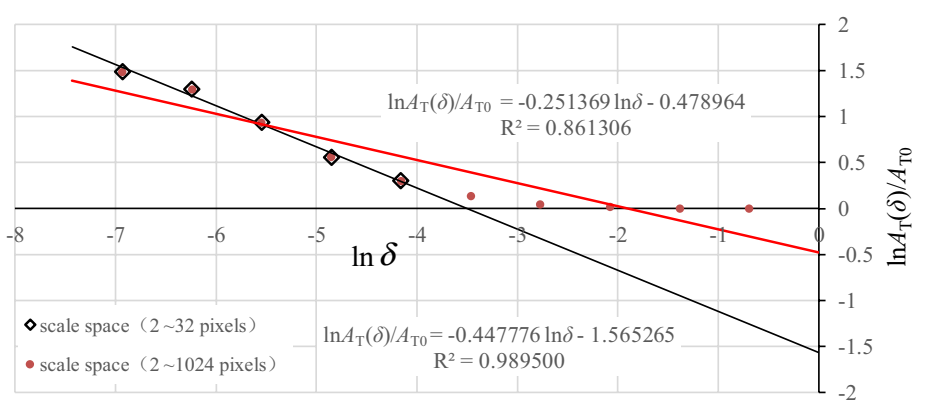

Fig. 10 Comparison diagram of fractal dimension fitting for the same surface at different scales 


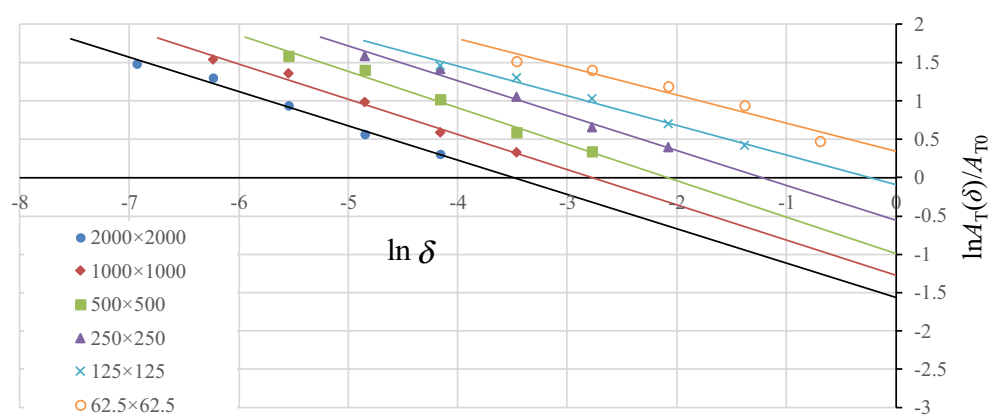

Fig. 11 Comparison diagram of fractal dimension fitting in different size

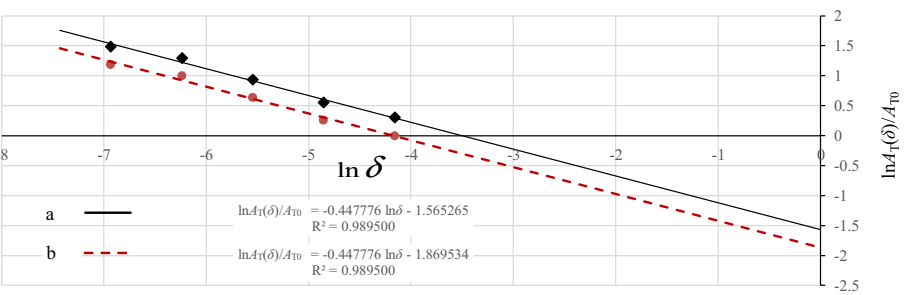

Fig. 12 Comparison diagram of the same fractal dimensions but intercept

About the Fig. 4 surfaces, the trends of the parameter $\Delta D_{\max }^{S D}$ are shown in Fig. 9. From Fig. 9, in general, the parameter $\Delta D_{\max }^{S D}$ decreases to the stable value as the structure surface size increases. Theoretically speaking, when the parameter $\Delta D_{\max }^{S D}$ equals to 0 , it can be considered that morphology features of the studied surfaces are nearly the same, and this size can be considered as the standard size of structural planes. In fact, it is difficult to find the two structural surfaces which the parameter $\Delta D_{\max }^{S D}$ equals to 0 due to the complex morphology of natural structural surface. So, we can analyze the trend of the parameter $\Delta D_{\max }^{S D}$ and find the stable value, and then, the corresponding size is the standard size of structural planes. From Fig. 9, to the surface of Fig. 4a, there is no stable value of the $\Delta D_{\text {max }}^{S D}$, but to the surface of Fig. $4 \mathrm{~b}$, we think the parameter $\Delta D_{\max }^{S D}$ tend to be stable when the size is $250 \mathrm{~mm} \times 250 \mathrm{~mm}$.

\section{Discussion}

\section{Determination of scale space for fractal dimension}

The scale space has an important influence on the calculation result of fractal dimension. Take the Fig. $4 \mathrm{~b}$ structural surface for example. If the scale space range is from 2 to 1024 pixels, there are 10 pairs of $\left\{\ln \left[A_{\mathrm{T}}(\delta) / A_{\mathrm{T} 0}\right], \quad \ln \delta\right\}$ to fit the fractal dimension, and the result is 2.251369 . When the scale space range is from 2 to 32 pixels, there are 5 pairs of $\left\{\ln \left[A_{\mathrm{T}}(\delta) / A_{\mathrm{T} 0}\right], \ln \delta\right\}$ to fit the fractal dimension, and the result is 2.447776 . The fitting results are shown in Fig. 10.

From the above analysis, we know the calculating results of fractal dimension are different with the different scale space. So, we investigate the size effect of structural 
surfaces using fractal dimension method, the uniform scale space should be adopted. Otherwise, there is no comparison. In this paper, because the minimum structure surface is $62.5 \mathrm{~mm} \times 62.5 \mathrm{~mm}$ ( 65 pixels $\times 65$ pixels), it's maximum scale space is 32 pixels. So, in this article, in order to make the calculating results comparable, the scale space range is set from 2 to 32 pixels.

\section{Determination of the parameter $\Delta D_{\max }^{S D}$ value}

Theoretically, when the parameter $\Delta D_{\max }^{S D}$ value is 0 , it can be considered that the fractal surfaces are the same morphological features. And, this size can be seen as the standard size of structural surfaces. However, it is difficult to find such standard size with the same fractal dimension in different regions due to the complex morphology of natural structural surfaces. Therefore, it is necessary to determine the parameter $\Delta D_{\max }^{S D}$ by not only the variation trend but also studying the mechanical properties.

\section{The characteristics of the parameter intercept relating with fractal dimension}

As shown in Fig. 10, for the same structural plane, the parameter intercept relating with fractal dimension is different as different scale space by PCM. But, to different sizes structural planes, even if the scale space is the same, the intercept varies greatly, as shown in Fig. 11. The Fig. 11 is the fitting results of the Case 2 of the Fig. $4 \mathrm{~b}$ with different sizes but the same scale space which is from 2 to 32 pixels. As can be seen from Fig. 11, the larger size of the structure surface, in order to get the fitting points in small scale space, the smaller $\ln \delta$ value is. So, the intercept is smaller.

Fractal dimension has been widely recognized by many scholars to describe the morphological characteristics of structural surfaces. And some scholars have tried to use the parameter intercept to describe the size effect characteristics of structural surfaces [10]. However, from the above analysis, there is a defect in describing the structural surface by the parameter intercept. For Fig. 11, the intercept value of the surface $62.5 \mathrm{~mm} \times 62.5 \mathrm{~mm}$ is much larger than that of the $2000 \mathrm{~mm} \times 2000 \mathrm{~mm}$. The main reason for this result is that the fitting points are distributed different location in the horizontal axis, rather than mainly depending on the morphology features.

Fortunately, in the following cases, the intercept can be used to further distinguish the roughness of structural surfaces, as shown in Fig. 12. The Line $b$ in the Fig. 12 is formed by the overall downward shift of 0.304269 of the Line a. Although the slope of the two lines is the same, that is, the fractal dimensions are the same, the values of $\ln \left[A_{\mathrm{T}}(\delta) / A_{\mathrm{T} 0}\right]$ on Line $b$ are all smaller than the values of that on Line $a$. Therefore, it can be concluded that the structural surface corresponding to Line $a$ is rougher than that corresponding to Line $b$. In this case, the roughness of structural surfaces $a$ and $b$ can be further distinguished by the parameter intercept. 


\title{
Conclusion
}

In this article, considering the shortcoming of the projective covering method, we put forward the improved projective covering method. Based on this, we analyzed the size effect characteristics of the two structural planes. By comparing and analyzing the fractal dimension trend of different size structural planes under different division schemes, the following conclusions are drawn.

1. Considering probability, by random connection method, the thousands of small grid cells in the whole structure plane are more consistent with the actual morphology. From the calculations, the fractal dimension by PCM is almost the limit value. So, it will be more reasonable that the mean of fractal dimension samples by IPCM is taken as the accurate fractal dimension of structure surfaces.

2. The size effect of structural surfaces roughness is related to the morphological characteristics of structural surfaces. Generally, as the size increases, the roughness of the structure surface first increases and then decreases. That is, it shows positive size effect in the small size range and negative size effect in the large size range.

3. Different size division schemes have an important influence on the size effect law of structural surfaces. In other words, the size division schemes of the same structural surface are different, and the size effect characteristics are also different. Therefore, the study of the size effect of structural surfaces cannot be limited to a certain size division scheme. Otherwise, the results are one-sided.

4. With the increase of the size of structural surfaces, the parameter $\Delta D_{\max }^{S D}$ generally decreases and gradually tends to be stable. The stability value can be used to determine the reasonable size of structural planes. This parameter provides a new way to determine the reasonable size of structural surfaces.

\begin{abstract}
Abbreviations
$A_{i}$ : Probability event (dimensionless); $A_{k}(\delta)$ : Area of the kth cell square $\left(\mathrm{mm}^{2}\right) ; A_{T 0}$ : Apparent area of the rough surface $\left(\mathrm{mm}^{2}\right) ; A_{T}(\delta)$ : Entire area of the rough surface under a certain scale measurement $\left(\mathrm{mm}^{2}\right)$; $D$ : Fractal dimension (dimensionless); $D_{A} S D$ : Average of fractal dimensions of the same size structure surface but from different area (dimensionless); $f_{i}$ : Counts of samples in the ith subset (dimensionless); $h_{a k} h_{b k} h_{c k}$ and $h_{d k}$ : Heights of a fracture surface at points $a, b$, $c$ and $d$, respectively ( $\mathrm{mm}$ ); IPCM: Improved projective covering method (dimensionless); $k$ : Number of valid subsets (dimensionless); $n$ : Number of samples (dimensionless); N: Preset number of samples (dimensionless); N ( $\delta$ ): Total number of cells with scale of $\delta \times \delta$ needed to cover the rough surface (dimensionless); P $\left(A_{j}\right)$ : Probability value of the event $A_{i}$ (dimensionless); PCM: Projective covering method (dimensionless); $r$ : Degrees of freedom (dimensionless); $x^{2}$ : Statistical variable (dimensionless); $\triangle$ : Distance of the adjacent subsets (dimensionless); $\Delta D_{\max } S D$ : Maximum difference of fractal dimensions of the same size structure surfaces but from different area (dimensionless); $\delta$ : Step size (dimensionless).
\end{abstract}

\section{Acknowledgements}

The authors would like to express sincere gratitude to all the anonymous reviewers for their comments devoted to improving the quality of our paper. This paper is funded by National Natural Science Foundation of China (51564038) and Excellent youth fund of Inner Mongolia university of science and technology (2016YQL04). These supports are gratefully acknowledged.

Authors' contributions

All authors read and approved the final manuscript.

Competing interests

The authors declare that they have no competing interests.

Author details

${ }^{1}$ Institute of Mining Research, Inner Mongolia University of Science and Technology, Baotou, Inner Mongolia 014010, People's Republic of China. ${ }^{2}$ School of Resources and Civil Engineering, Northeastern University, Shenyang, Liaoning 110819, People's Republic of China. 
Received: 23 August 2019 Accepted: 24 August 2020

Published online: 19 November 2020

\section{References}

Ai T, Zhang R, Zhou HW, Pei JL (2014) Box-counting methods to directly estimate the fractal dimension of a rock surface. Appl Surf Sci 314:610-621

Bahaaddini M, Hagan PC, Mitra R, Hebblewhite BK (2014) Scale effect on the shear behaviour of rock joints based on a numerical study. Eng Geol 181:212-223

Barton N, Bandis S (1982) Effects of block size on the shear behaviour of jointed rock. In: Proceedings of the $23 \mathrm{rd}$ US rock mechanics symposium, Berkeley, CA, pp 739-760

Belem T, Homand-Etienne F, Souley M (2000) Quantitative parameters for rock joint surface roughness. Rock Mech Rock Eng 33(4):217-242

Buzzi O, Casagrande D (2018) A step towards the end of the scale effect conundrum when predicting the shear strength of large in situ discontinuities[J]. Int J Rock Mech Min Sci 105:210-219

Chen SJ, Zhu WC, Yu QL, Liu XG (2016) Characterization of anisotropy of joint surface roughness and aperture by variogram approach based on digital image processing technique. Rock Mech Rock Eng 49(3):855-876

Clarke KC (1986) Computation of the fractal dimension of topographic surfaces using the triangular prism surface area method. Comput Geosci 12(5):713-722

Cravero, M, labichino, G, Ferrero, AM (2001) Evaluation of joint roughness and dilatancy of schistosity joints. In: Sarkka P, Eloranta P (eds) Rock mechanics-a challenge for society; proceedings of Eurock 2001, Espoo, Finland, 4-7 June 2001. A.A. Balkema, Rotterdam, pp. 217-222

El-Soudani SM (1978) Profilometric Analysis of Fractures. Metallography 11(3):247-336

Fardin N, Feng Q, Stephansson O (2004) Application of a new in situ 3D laser scanner to study the scale effect on the rock joint surface roughness. Int J Rock Mech Min Sci 41(2):329-335

Fardin N (2008) Influence of structural non-stationarity of surface roughness on morphological characterization and mechanical deformation of rock joints. Rock Mech Rock Eng 41(2):267-297

Fardin N, Stephansson O, Jing L (2001) The scale dependence of rock joint surface roughness. Int J Rock Mech Min Sci 38(5):659-669

Ge YF, Kulatilake PHSW, Tang HM, Xiong CR (2014) Investigation of natural rock joint roughness. Comput Geotech 55:290-305

Grasselli G, Wirth J, Egger P (2002) Quantitative three-dimensional description of a rough surface and parameter evolution with shearing. Int J Rock Mech Min Sci. 39(6):789-800

Jang HS, Kang SS, Jang BA (2014) Determination of joint roughness coefficients using roughness parameters. Rock Mech Rock Eng 47(6):2061-2073

Jiang Y, Li B, Y. Tanabashi Y, (2006) Estimating the relation between surface roughness and mechanical properties of rock joints. Int J Rock Mech Min Sci 43(6):837-846

Kwaśny W (2009) A modification of the method for determination of surface fractal dimension and multifractal analysis. J Achiev Mater Manuf Eng 33(2):115-125

Leal-Gomes MJA (2003) Some new essential questions about scale effects on the mechanics of rock joints. In: Handley M (ed) Proceedings of the 10th ISRM Congress: Technology roadmap for rock mechanics. Sandton, South Africa, South African Institute of Mining and Metallurgy, pp. 721-727

Li J, Du Q, Sun C (2009) An improved box-counting method for image fractal dimension estimation. Pattern Recogn 42(11):2460-2469

Liu Y, Che LY, Wang HM, Jiang LL, Zhang Y, Zhao JF, Wang DY, Zhao YC, Song YC (2014) An improved differential box-counting method to estimate fractal dimensions of gray-level images. J Vis Commun Image Represent 25(5):1102-1111

Mughieda O, Alzo'ubi AK, (2004) Fracture mechanisms of offset rock joints - a laboratory investigation. Geotech Geol Eng 22(4):545-562

Myers NO (1962) Characterization of surface roughness. Wear 5(3):182-189

Ohnishi Y, Yoshinaka R (1992) Laboratory investigation of scale effect in mechanical behaviour of rock. In: Myers LR, Tsang CF, Cook NGW, Goodman RE (eds) Proceedings of the conference on fractured and jointed rock masses, Lake Tahoe, CA, 3-5 June 1992. A.A. Balkema, Rotterdam, pp. 465-477

Sheng Z, Xie SQ, Pan CY (2002) Probability and statistics. China Higher Education Press, Beijing

Tatone BSA, Grasselli G (2010) A new 2D discontinuity roughness parameter and its correlation with JRC. Int J Rock Mech Min Sci 47(8):1391-1400

Tatone BSA, Grasselli G (2013) An investigation of discontinuity roughness scale dependency using high-resolution surface measurements. Rock Mech Rock Eng 46(4):657-681

Tse R, Cruden DM (1979) Estimating joint roughness coefficients. Int J Rock Mech Min Sci Geomech Abstr 16(5):303-307

Yong R, Ye J, Li B, Du SG (2018) Determining the maximum sampling interval in rock joint roughness measurements using Fourier series. Int J Rock Mech Min Sci 101:78-88

Yu XB, Vayssade B (1991) Joint Profiles and their Roughness Parameters. Int J Rock Mech Min Sci Geomech Abstr 28(4):333-336

Wen XJ, NinaS.-N L, (2009) An improved algorithm for computing local fractal dimension using the triangular prism method. Comput Geosci 35(6):1224-1233 
Xie HP, Wang JA (1999) Direct fractal measurement of fracture surfaces. Int J Solids Struct 36(20):3073-3084

Xie HP, Wang JA, Stein E (1998) Direct fractal measurement and multifractal properties of fracture surfaces. Phys Lett A 242(1-2):41-50

Zhou HW, Xie HP (2003) Direct estimation of the fractal dimensions of a fractures surface of rock. Surf Rev Lett 10(5):751-762

Zhang YH, Zhou HW, Xie HP (2005) Improved cubic covering method for fractal dimensions of a fracture surface of rock. Chin J Rock Mechan Eng 24(17):3192-3196

\section{Publisher's Note}

Springer Nature remains neutral with regard to jurisdictional claims in published maps and institutional affiliations.

Submit your manuscript to a SpringerOpen ${ }^{\circ}$ journal and benefit from:

- Convenient online submission

- Rigorous peer review

- Open access: articles freely available online

- High visibility within the field

- Retaining the copyright to your article

Submit your next manuscript at $\gg$ springeropen.com 